\title{
Universidad e investigación para el bien común: la función social de la Universidad
}

\author{
Enrique Javier Díez Gutiérrez (*)
}

(*) Universidad de León - España

\begin{abstract}
RESUMEN
En un Estado Social y Democrático la universidad ha de orientarse a la prestación de un servicio público de generación de conocimiento en el que se contemplen los intereses generales de la ciudadanía, el bien común, funcionando como un agente de cambio y mejora social. Pero nuestras instituciones de educación superior, siguiendo lógicas globales neoliberales, promovidas por organizaciones económicas internacionales, se están transformando progresivamente en "universidades corporativas o emprendedoras" dominadas por una cultura empresarial interesada sobre todo en lograr posiciones sobresalientes en rankings internacionales. Es hora de defender un modelo de universidad pública al servicio de las necesidades sociales reales y prioritarias y no solo las demandas del mercado. Pero también es necesario profundizar y priorizar la función social de la universidad, consiguiendo un contrato con la sociedad en su conjunto, que entienda la universidad no solo como institución académica de prestigio, sino como un bien público y un agente dinamizador para la transformación social. Este modelo social de Universidad debería basarse en el refuerzo del compromiso y la responsabilidad social de las universidades públicas, la vinculación con los sectores sociales más desfavorecidos y el compromiso mutuo en pos de la justicia social.
\end{abstract}

Palabras Clave: Educación Superior, Capitalismo académico, Función social de la Universidad, Bien Común, Servicio Público.

\section{University and research for the common good: the social function of universities}

\section{ABSTRACT}

In a social and democratic state, universities must strive to provide a public service, generating knowledge of general interest to citizens for the common good and working as agents of social change and improvement. However, in line with the global neoliberal logic promoted by international economic organisations, universities are increasingly metamorphosing into "corporate or entrepreneurial institutions" dominated by a business culture primarily interested in achieving a high international ranking. It is time to defend the model of a public university in the service of real, pressing social needs rather than market demands. It is also necessary to prioritise and expand the social function of universities, establishing a contract with society as a whole that considers the university not only as a prestigious academic institution but also as a common good and a dynamic agent for social change. This social model of the university should be based on strengthening public universities' social responsibility and commitment, ties with the most disadvantaged sectors of society and a mutual commitment to social justice.

Keywords: Higher education, Academic capitalism, Social function of universities, Common good, Public service.

"Nuestra universidad no puede cumplir su misión sin una confluencia de competencia técnica y compromiso político" (Ellacuría, 1999, 100).

Según nos indica el artículo primero de la Ley Orgánica de Modificación de la Ley Orgánica de Universidades (LOMLOU), la actual ley que rige la educación superior, las universidades son instituciones que deben cumplir tres misiones fundamentales: la enseñanza y formación superior; la investigación y generación de conocimiento; así como el servicio a la sociedad mediante el más amplio impacto cultural, social y económico de su actividad en la construcción de una sociedad mejor, más justa y democrática.

Los Estatutos de una Universidad, como por ejemplo la de Valladolid, así lo recogen en su art. 3: "La Universidad de Valladolid, como expresión de su compromiso con la sociedad, está al servicio de su progreso intelectual y material y asume como valores inspiradores de su actividad la promoción de la paz y de la cooperación entre los pueblos, del desarrollo sostenible, y de la igualdad de género y de oportunidades. Asimismo, los principios de igualdad, libertad, justicia, solidaridad y democracia interna, junto a los demás que emanan de la Constitución, orientan el cumplimiento de sus fines." 
Sobre la base de esas misiones y de su propia concepción, en un Estado que se caracteriza como Social y Democrático, la universidad ha de orientarse por tanto a la prestación de un servicio público de generación de conocimiento en el que se contemplen los intereses generales de la ciudadanía, el bien común, funcionando así como un agente de cambio y mejora social.

Es más, desde la Universidad nos deberíamos plantear como uno de los objetivos fundamentales de la educación superior, como proponen Nichols \& Berliner (2007): “ser el número uno en el mundo en porcentaje de jóvenes que están política y socialmente implicados. Mucho más importante que nuestras puntuaciones en matemáticas y nuestras puntuaciones en ciencia es la implicación de la generación siguiente en el mantenimiento de una democracia real y en la construcción de una sociedad más justa para los que más la necesitan: los jóvenes, los enfermos, los ancianos, los parados, los desposeídos, los analfabetos, los hambrientos y los desamparados. Se deberían identificar los centros que no pueden producir ciudadanía politicamente activa y socialmente útil y divulgar sus tasas de fracaso en los periódicos".

Sin embargo, no parecen ser estas las orientaciones y principios que las universidades y las políticas públicas sobre la educación superior estén alentando actualmente. No tenemos más que recordar el escándalo del denominado "mastergate" que implicó a tantos políticos en la Universidad Rey Juan Carlos, efecto colateral del modelo neoliberal instaurado con el denominado "Plan Bolonia" que ha convertido los dos últimos años de las carreras universitarias (los másteres) en un lucrativo negocio, sobre todo para las universidades privadas, y cuyas consecuencias empiezan a aflorar actualmente a través de los escándalos que aparecen en los medios de comunicación sobre los másteres.

\section{Universidad Neoliberal}

La ideología neoliberal está dominando cada vez más la dinámica de las instituciones universitarias. Nuestras instituciones de educación superior, siguiendo lógicas globales promovidas por organizaciones económicas internacionales (FMI, BM, OMC, OCDE), se están transformando progresivamente en "universidades corporativas o emprendedoras" dominadas por una cultura empresarial interesada sobre todo en lograr posiciones sobresalientes en rankings internacionales. Lógicas que se han instalado en la agenda pública hasta el punto de que se asumen como propias por las propias instituciones universitarias y sus comunidades académicas y, por lo tanto, se convierte en legítimas y legitimadas por los propios protagonistas (Pineda \& Celis, 2017).

La universidad está orientándose de manera creciente a la formación para el mercado laboral, con un doble modelo: de élite para quienes se lo pueden pagar y de auténtico "ejército de reserva del precariado" (Sevilla, 2010; Fernández, Urbán y Sevilla 2013) para quienes no tienen recursos y medios. Sir Nigel Thrift, el impulsor de los cambios neoliberales en la universidad británica, cuyo lema "no hay dinero, no hay dinero, no hay dinero" se hizo popular entre los gestores, reconoce que las universidades en los puestos superiores de los rankings, aunque les convenga hacer ver que no son elitistas a través de algunas medidas que ayuden a paliar esa deficiencia, "producen élites", formando parte de la cadena de la desigualdad y sus beneficios provienen de seguir practicando ese juego.

Por eso los estudiantes de clase media están inmersos en una carrera continua para ser admitidos en los mejores centros. Han emprendido lo que Thrift (2017) llama "carrera armamentística educativa" por situarse bien en el futuro: les pagan clases particulares a sus vástagos, les pagan todo tipo de actividades extraaca- démicas, les envían a universidades en el extranjero, se mudan a zonas con centros prestigiosos, y se oponen firmemente a que otra clase de estudiantes accedan a ellos para no perder posibilidades. Esa inversión se refuerza con los posgrados y con las prácticas no remuneradas, casi reservadas en exclusiva a quienes pueden subsistir sin trabajar.

Las clases acomodadas saben que es mucho más difícil que antes que el sistema formativo sea una vía de ascenso social, que los puestos de élite van a ser menos en el futuro y que quienes no estén situados en los estratos adecuados no van a tener las mismas oportunidades, por lo que están educando a sus hijos e hijas para que aprendan idiomas con el acento correcto, se formen en centros de élite que les permitan el acceso a los espacios que les aseguren prestigio y relaciones (Thrift, 2017). Esta competición darwinista deja pocas puertas abiertas a quienes no estén ya en la élite.

De esta forma la universidad refuerza su poder jerarquizador. Sólo unas pocas personas pueden acceder a ellas, con la cada vez mayor subida de tasas académicas que se ha impuesto en todo el mundo, sobre todo a los tramos superiores de másteres, ya que no todas tendrán los recursos económicos suficientes para hacer frente a estas elevadísimas tasas de matriculación, a los desplazamientos y el alojamiento. Funcionan así como "clubes", cuyos exclusivos socios van a poder disfrutar de toda una serie de ventajas que les reforzarán todavía más su situación como élite.

Sabemos que estas medidas tienen como objetivo evitar que una parte de la población pueda cursar estudios universitarios, precisamente la parte de la población que menos recursos tiene. Pero la ideología neoliberal no solo trata de excluir a esa parte de la población que ha sido capaz de acceder a la Educación Superior en estas últimas décadas, sino que trata también de incluir a la parte de la población que queda en las aulas universitarias en lo que podríamos denominar "conciencia de clase neoliberal", mediante mecanismos cotidianos, sutiles y pertinaces que "normalizan" esa ideología, que se aproximan a lo que Rushkoff (2000) denomina "coerción", mediante estrategias biopolíticas de gubernamentalidad, de "gobierno-por-la-mente" (Foucault, 1975), a través del gobierno por la deuda.

La creación de una burbuja universitaria es uno de estos mecanismos que ya se está implantando en nuestro país, siguiendo el modelo de EE. UU., donde la burbuja de los préstamos estudiantiles asciende a más de 1,2 billón de dólares de deuda (equivale a lo que produce la economía española, rusa o australiana en un año), aunque, como siempre en la práctica neoliberal, el Estado es el garante de la deuda en última instancia. Ya se ha producido el primer embargo judicial a una estudiante por no devolver un préstamo renta de estudios (Barrón, 2018).

Los que consiguen acceder tienen que pagar, cada vez más, con cada vez menos dinero para estudios que son cada vez más largos (Delapierre, 2015), convirtiéndolos en precariado endeudado desde que empiezan sus estudios universitarios. Es lo que Harvey (2014) denomina acumulación por desposesión. Ese endeudamiento cambia la lógica y la relación de los estudiantes con los estudios y con su formación, generando lo que se denomina "deudocracia universitaria": el gobierno por la deuda de las mentes, la orientación de los deseos, las elecciones, los comportamientos, las aspiraciones y el futuro de los estudiantes universitarios.

Mediante este tipo de mecanismos se disciplina a los estudiantes para que entiendan que deben optar por los estudios más "rentables" que les permitan devolver la deuda, pero además naturaliza el sistema de mercado y endeudamiento como algo natural, inevitable e implacable y enseña a asumirse como 
"persona deudora", sometida a un eterno ciclo de "vivir pagando para morir debiendo".

Esto anula el sustrato común de cooperación y solidaridad entre los estudiantes, que tienen que competir para conseguir las mejores posibilidades de rentabilidad de su "inversión educativa", al tiempo que fragmenta y culpabiliza aún más a esos miles de jóvenes que asumen que el fracaso o el éxito depende solo de sus propias capacidades (Fernández \& Valdés, 2015).

Michel Foucault (1975), señalaba que las sociedades occidentales actuales han abandonado el modelo disciplinario y, en contraste, han adoptado herramientas de control social que requieren la participación de los involucrados. La ideología neoliberal nos educa para "elegir libremente", incluso desear, pertenecer a su engranaje. La nueva explotación es amada. El "opio del pueblo" es el propio sistema. Esta ideología neoliberal se ha convertido así en una "racionalidad" que se orienta a la conducción de las conductas (Foucault, 2004). Al igual que los organismos financieros internacionales gobiernan a los pueblos y las naciones a través de la deuda, los bancos y financieras privadas gobiernan a los estudiantes también utilizando una deuda, que no prescribe, a lo largo de su vida.

\section{Capitalismo académico}

Los programas de estudio y las investigaciones se están definiendo en función de la rentabilidad esperada en el mercado (Garzón, 2015, 7). El espacio público está siendo apropiado y rentabilizado por las marcas comerciales. Los bancos erigen sus "templos" del comercio como nuevas catedrales en el centro de los campus universitarios. La Nueva Gestión Pública se implanta también en la organización de la docencia y en la gestión de la ciencia. La universidad se ha convertido en una gran empresa que hace "caja" por casi todo y ya no entiende de compartir libremente el saber y el conocimiento.

La doctrina neoliberal aplicada a la enseñanza superior, en un contexto de recorte presupuestario continuado, conduce a la explosión del denominado "capitalismo académico" (Readings, 1996; Slaughter \& Leslie, 1997; Anderson, 2001; Johnson, Kabanagh \& Mattson, 2003; Bok, 2003; Gould, 2003; Slaughter \& Rhoades, 2004; Fernández, 2009; Rhoades \& Slaughter, 2010; Massé, 2014; Pineda \& Celis, 2017). Universidades cada vez más comprometidas en una competencia de tipo comercial, en busca de fuentes de financiación complementarias y con una formación y docencia fundamentalmente orientada por las competencias pre-identificadas por el mercado de trabajo. Otras capacidades que podrían promover una sociedad más justa y mejor van quedando "obsoletas" y se las obvia progresivamente (Barnett, 2001). La asignación presupuestaria ya no se hace en función de las necesidades de la institución para su mejora, sino según los resultados obtenidos en rankings estandarizados y contratos definidos por la consecución de objetivos relacionados con esos rankings. Mientras, se recorta el presupuesto para proyectos "improductivos" de orientación humanística y/o crítica.

Se le asigna cada vez más el aval institucional a lo que es considerado como económicamente útil. La universalidad propia del conocimiento universitario se reorienta hacia un modelo pragmático e instrumental del saber al servicio de la economía, formando a los estudiantes universitarios como futuros trabajadores y trabajadoras de empresas a costa del dinero público e investigando en función de las demandas de las empresas.

Lo que se ha convertido en la tarea esencial de la enseñanza superior en la época de la globalización neoliberal, ya no es la producción de 'seres humanos razonables', es decir, de personas capaces de acceder, analizar y juzgar, razonable y rigurosamente, el conocimiento y la ciencia, aportando a la comunidad científica y social nuevos avances para mejorar la vida de la comunidad, sino la de asalariados técnicamente cualificados y adaptados a las exigencias de las demandas empresariales cambiantes en función del constante fluctuar de los nichos de mercado donde obtener mayor rentabilidad. El ideal de una formación humanista que pretendía la emancipación intelectual y el desarrollo de un ser humano integral, para quien el trabajo no constituía la exclusiva ocupación de la vida, se ha quebrado en la era neoliberal (Laval, 2004).

La universidad ya no es más un lugar tranquilo para enseñar, realizar trabajo académico, investigar y debatir sobre la ciencia y el conocimiento a ritmo pausado y profundo, sino que es una gran maquinaria, un potente negocio, una gran empresa competitiva que requiere la obtención de resultados cuantificables, que puedan ser patentados, transferidos y explotados y que procuren una buena colocación en los índices de productividad investigadora y permitan estar en la parte alta del ranking de Shanghái (el Academic Ranking of World Universities, habitualmente conocido como ranking de Shanghái), del ranking del CSIC o de cualquier otro. Ahora las universidades ofrecen sus instalaciones científicas y su credibilidad académica para que las empresas las utilicen a cambio de esa financiación que ha sido suprimida por los gobiernos (Hirtt, 2003). Las empresas "donantes" imponen sus logotipos en las paredes y el mobiliario, vuelven a bautizar edificios y los espacios colectivos con sus marcas, patrocinan cátedras académicas y de investigación a cambio de una denominación que revela el origen de los fondos.

La investigación que proviene de estas cátedras, lógicamente, responden a los intereses de quienes las patrocinan, no sólo porque es ante quienes hay que demostrar la eficacia de su inversión a través de resultados "tangibles" y que generen "beneficios", sino también porque recortan y definen los temas e intereses de las investigaciones posibles, así como las prioridades de estas. En algunos casos incluso, los fondos aportados por la parte privada limitan abiertamente la libertad de pensamiento y la reflexión crítica, con cláusulas de confidencialidad y de exclusividad, que implican el derecho de impedir o aplazar la publicación de los estudios, si éstos entran en contradicción o suponen un cuestionamiento de los intereses o la imagen de la empresa que los financia.

De esta forma, la 'disciplina por el dinero' que se impone en el mundo universitario, introduce muy serias amenazas en la vida intelectual y el pensamiento académico. Y las políticas públicas de los gobiernos conservadores y neoliberales profundizan en este sentido. De hecho, la investigación promovida por la administración pública en España se orienta cada vez más hacia una clara privatización.

\section{Privatización de la investigación}

El "Plan Estatal de Investigación Científica y Técnica y de Innovación (2017-2020)", que es la principal herramienta española para el desarrollo y la promoción de la investigación científica, privatiza la investigación pública en España. Se orienta principalmente a financiar la investigación en empresas privadas y el "liderazgo empresarial" de la investigación pública.

En un contexto de recortes de financiación pública en la investigación, donde España "es el país que más recortes ha realizado en este campo" (Molero \& de No, 2015; Corral, 2016; Mediavilla, 2017), mientras, en plena crisis, la inversión en investigación en Alemania ha crecido un 38\% y la de Reino Unido, un $39 \%$ (Sampedro, 2017), la privatización neoliberal de la I+D se produce, no solo estrangulando la financiación pública, sino 
también y simultáneamente introduciendo el control de la gran empresa privada sobre este ámbito también.

Las convocatorias de financiación de I+D en determinadas Comunidades Autónomas excluyen de facto la investigación en ciencia básica así como en ciencias jurídicas, económicas y sociales además de humanidades, al establecer que "solamente tendrán financiación pública aquellos proyectos de investigación en ámbitos determinados" (RIS3), que ha supuesto que hayan sido excluidos el $80 \%$ de los proyectos presentados relacionados con Arqueología, Química inorgánica, Educación, Historia, Literatura, Derecho o Patrimonio.

Pero esto se ve agravado cuando condicionan, además, la financiación pública a la previa obtención de financiación privada, hasta el punto de llegar a convertir esta exigencia en un sorprendente requisito de calidad. Lo cual transforma el sentido y la finalidad de la investigación, porque buena parte de la investigación, especialmente la que no tiene inmediato rendimiento mercantil traducido en patentes o resultados comercializables, no parece susceptible de suscitar interés para el modelo empresarial predominante, que presume de buscar el beneficio rápido e inmediato de los accionistas.

Además, porque implica estrangular la financiación y suprimir de facto investigaciones fundamentales relacionadas con cuestiones regionales y locales de interés para la gente empobrecida, las minorías o las mujeres de clase trabajadora de las zonas rurales, por ejemplo. No parece que las empresas estén interesadas y dispuestas a aportar fondos para esas temáticas. Las prioridades de la investigación ya no son, pues, generar las bases para construir un modelo social más justo en lo económico, más sabio en lo social y más ecológico en lo natural.

Si a esto añadimos lo que ya se denomina, de forma irónica y a la vez sarcástica, "la loca carrera investigadora" del profesorado universitario (Jorge Alonso \& De Frutos, 2016-2017), parece que las prioridades han cambiado sustancialmente.

Para acceder y para progresar en la carrera académica, la labor investigadora de todo el profesorado universitario es valorada en función de unos "índices de impacto" de publicaciones en revistas controladas por un duopolio privado, gestionado por dos multinacionales (Thomson Reuters, ahora Clarivate Analytics y Elsevier) que lo han convertido en un ingente negocio a costa del dinero público (Buranyi, 2017).

Controlar el acceso a la literatura científica es como controlar la ciencia. Ya no se trata tanto de qué se ha descubierto con la investigación, sino en qué revista se consigue publicar dicha investigación y qué artículos han sido más mencionados. La "visibilidad" traducida en "ser citado" se ha convertido en la "moneda" de cambio del mundo científico (Jorge Alonso \& De Frutos, 2016-2017). Lo cual consolidan la competitividad, la cuantificación y la comparación del "impacto por citación" como prueba "objetivable" de la calidad del trabajo de investigación (Amigot \& Martinez, 2015). La ciencia se ha convertido así "hoy en día en una mercancía: está en el mercado y se paga un precio por ella" (Mirowski, 2011; Saltelli, 2017; Monbiot, 2017).

Ha sido decisión política de los gobiernos el que se haya supeditado la evaluación de la carrera profesional universitaria a la publicación en revistas incluidas en los índices de estas dos multinacionales (Buranyi, 2017; Munárriz, 2017). "Se ha llegado a un punto en que, para muchos ámbitos científicos y profesores, fuera de las revistas indexadas no hay vida" (Ramió, 2014, 117). Publicar o perecer. Aunque Alemania se plantó en 2017 y se negaron a seguir pagando y avanzan en un modelo de 'open access', que les está funcionando (Villareal \& Escudero, 2018).

Para las universidades tampoco es fácil escapar a esta presión, ya que sus posiciones en los rankings internacionales pasan en buena parte por la cantidad de publicaciones en estas revistas de alto impacto que logra su profesorado. Revistas cuya gran mayoría pertenece a países anglosajones y tienden a citar a sus compatriotas (Flores \& Villaseñor, 2015); centradas en análisis y metodologías que interesan a los anglosajones con relación a su realidad; que obligan a referenciar un tipo de literatura muy alejada de la realidad no anglosajona renunciando a otros objetos, marcos teóricos y metodologías más apropiados para el análisis de la propia realidad y contexto social (Ramió, 2014; Barsky, 2014).

El problema colateral es que la no publicación en estas revistas de "impacto" se "penaliza" con un aumento del tiempo de docencia. Esto ha consolidado un imaginario en el que la docencia parece tener un valor inferior. Por lo que una finalidad fundamental de la educación superior, la formación, pasa a considerarse casi como carga o "castigo" (Rodríguez Victoriano, 2017).

\section{Una universidad para el bien común}

La primera medida necesaria, si queremos consolidar otro modelo de universidad posible al servicio del bien común, es revertir la privatización de la Educación Superior. Como demuestra el informe bianual 2014-2015 de la Conferencia de Rectores de las Universidades Españolas (CRUE), titulado "La Universidad Española en cifras", entre 2008 y 2014 las universidades públicas han experimentado un retroceso en el total de estudiantes (del $89,2 \%$ al $85,3 \%$ ), mientras que las privadas han aumentado (del $10,9 \%$ al $14,7 \%$ ), dado que las políticas del PSOE y del PP han incentivado la creación de universidades privadas en el Estado a razón de al menos una cada año.

Por el contrario, este informe confirma que el tamaño del sistema universitario español, no solo se encuentra dentro de parámetros de normalidad, sino que resulta inferior a los registrados en el panorama internacional de los países desarrollados industrialmente de la OCDE. De hecho, comprueba que España tiene una oferta de instituciones universitarias de un tamaño (por número de estudiantes) equiparable al de otros países continentales europeos. Lo que se está produciendo ahora es la sobresaturación de universidades-empresas privadas que están privatizando la educación superior.

Es necesario también revertir el ciclo de privatización que se está profundizando a través de la subcontratación de servicios de las universidades públicas, impuesta desde instancias políticas mediante normativas neoliberales que limitan la necesaria ampliación de plantillas. Servicios subcontratados que suelen conllevar peor y menor tiempo de prestación, pues el beneficio de las empresas que los gestionan se extrae de las condiciones laborales del personal que lo presta: eventualidad generalizada, mayor carga de trabajo, menor posibilidad de contestación... Así como eliminar la privatización que se produce también a través de fundaciones, justificadas por su supuesta mayor agilidad de gestión, pero que en la práctica sustraen al control democrático de la comunidad universitaria. Al igual que la creación de sociedades anónimas y spin off que pasan a prestar servicios propios de la universidad y que llevan a la hora de la verdad a imposibilitar una gestión transparente de los fondos públicos.

La segunda medida es reducir de forma inmediata los niveles de precios públicos universitarios hasta llegar a su completa gratuidad. El ministro Méndez de Vigo propuso en marzo de 2018 que la horquilla de precios que podrían aplicar las autonomías sería entre el $0 \%$ y el $25 \%$, lo que permite que las comunidades puedan convertir sus universidades en gratuitas. Es necesario recordar que la mejor forma de garantizar la igualdad de oportunidades y el derecho a la educación es la gratuidad de los estudios 
universitarios, de modo que en ningún caso se den discriminaciones por razón de capacidad económica.

Pero lo cierto es que España ha incrementado sus precios públicos siendo uno de los más elevados de la Unión Europea. Lo cual no ha venido acompañado de un aumento equiparable de las becas y ayudas al estudio, sabiendo que la principal barrera al acceso es la económica. Un alcance de ayudas de mucho menor cuantía que la de la mayoría de los países europeos: en los países escandinavos el porcentaje oscila entre el 100 y el 58\%; en Holanda, el 76\%; Irlanda, 47\%; Francia, 35\%; Alemania (25\%), Bélgica $(20 \%)$, Austria (15\%). Y en cuanto a la financiación media por persona que obtiene una beca (2.637 euros en el curso 2014-2015), se vuelve a niveles del curso académico 2006/2007, muy lejos de los 3.256 euros del curso 2012/2013.

La tercera medida es financiar las universidades públicas con un $2 \%$ del PIB, dado que han visto recortados sus presupuestos, en el período de 2010 a 2014, en 1.213 millones de euros, lo cual ha tenido una incidencia sustantiva en el normal desarrollo de la actividad universitaria; en la disminución de 7.500 empleos públicos (3.486 de personal docente e investigador y 4.068 de personal de administración y servicios), volviendo a plantillas universitarias de profesorado similar al que había a finales del año 2006, como asegura el Informe de la CRUE; y revertir igualmente los recortes en la financiación para la investigación, que desde el año 2008 ha supuesto un descenso del 24,6\% (mientras el porcentaje del PIB dedicado a I+D aumentaba un 35,7\% en Alemania entre 2009 y 2016). Y eso que, como dice irónicamente Martínez García (2017), "el diseño institucional de apoyo a la investigación está pensado dando por supuesto que los investigadores son delincuentes, y que solo mediante complejos trámites administrativos pueden demostrar su inocencia, por lo que, a la poca financiación, hay que añadir el exceso de burocracia".

Tenemos que cambiar estas políticas de recortes austericidas, que destinan nuestros recursos e impuestos a pagar el rescate de los bancos y grandes financieras. Es cuestión de voluntad política y decisión sobre a qué se destinan nuestros recursos. Si se quiere, se puede.

Una cuarta medida, complementaria de las anteriores, es erradicar de una vez por todas la precariedad del personal universitario e investigador, empezando de forma urgente por la figura del profesorado asociado, cuya pésimas condiciones laborales y precariedad son extremas, lo cual influye muy negativamente en la docencia y la calidad universitaria.

Más medidas que se han de tomar pasan por la eliminación de pruebas de acceso generales a la educación superior, pues la limitación del acceso de la población a los estudios superiores supone un freno a la democracia social y favorece la reproducción de las desigualdades y jerarquías sociales. Se debe favorecer el acceso del mayor número posible de población a la Enseñanza Superior, planificando con anterioridad el coste financiero y material que pueda suponer la ampliación del número, para evitar la masificación y el deterioro de las condiciones de enseñanza por limitaciones espaciales, económicas y de horario.

Promover la cooperación entre campus universitarios, entre facultades, equipos de investigación y docentes. Creando una cultura de colaboración en vez de competición, y generando un conocimiento abierto, libre y compartido que ayude a avanzar a la sociedad, en vez de promocionar carreras individuales basadas en la competencia y el trabajo individual. Se trata de defender una lógica de cooperación y de ayuda mutua en la Educación Superior.

Incluso se podría implantar un "impuesto de educación", pagado por las empresas que usen personal o conocimiento en el que ha habido inversión pública previa, puesto que gran parte del conocimiento y formación del personal cualificado se genera en nuestras universidades y centros de investigación públicos. Este "bien social" es aprovechado por las empresas y, por lo tanto, deberían contribuir con un retorno al Estado, y por ende a la sociedad en su conjunto, que permitiera recuperar parte de la inversión. Lo cual redundaría en una mejora del sistema educativo y científico, favoreciendo a todos, incluida a las empresas, ya que una mejora de la educación pública se traduciría en una mayor y mejor participación de personal cualificado y un acceso a conocimiento puntero (Moya, Pérez-Espona, Hortal, Valladares, Rodríguez-Gironés, Santamaría \& Montserrat, 2017).

La universidad requiere igualmente un autogobierno democrático y una gestión interna responsable. Las nuevas tendencias sociales apuntan cada vez más a métodos de trabajo en equipo, responsabilidad compartida y cooperación. La electividad de los cargos unipersonales y la colegialidad de los órganos de decisión son la mejor opción para esa corresponsabilización de la comunidad universitaria y para la transparencia que debe guiar todo el proceso. Por lo que los Consejos Sociales es imprescindibles reconvertirlos en órganos de representación de la comunidad social y no de la clase empresarial, como se han conformado actualmente, donde tengan más representación y poder de decisión movimientos sociales, colectivos vecinales, sindicatos, colectivos de investigación crítica, etc. Además, esa colegialidad conlleva reequilibrar la representación de todos los sectores universitarios en los órganos de gobierno e incrementar en particular el peso del estudiantado. Lo cual exige también un nuevo estatuto del estudiante más democrático.

Es hora de defender un modelo de universidad pública al servicio de las necesidades sociales reales y prioritarias y no solo las demandas del mercado. Pero también es necesario profundizar y priorizar la función social de la universidad, consiguiendo un contrato educativo con la sociedad en su conjunto, que entienda la universidad no solo como institución académica de prestigio, sino como un bien público y un agente dinamizador para la transformación social (Calella, 2013).

Este modelo social de Universidad debería basarse en dos conjuntos de principios fundamentales: por una parte, el refuerzo del compromiso y la responsabilidad social de las universidades públicas, manteniendo el principio de independencia académica; simultáneamente, la vinculación de la sociedad y de la universidad y el compromiso mutuo en pos de la justicia social es el pilar que debe vertebrar el funcionamiento de la institución académica superior (Manzano, 2011; Vila, 2012).

La universidad, como bien público de utilidad social, se debe a la sociedad en la que se ubica y esto exige un funcionamiento y una organización que permitan mantener el vínculo universidad-sociedad, uno de los pilares de la universidad pública con el que se busca favorecer la extensión y compartir el conocimiento universitario a todos los rincones de la sociedad.

Por tanto, las universidades deben crear estructuras que permitan la vinculación externa y permanente con la sociedad y los diferentes actores sociales y territoriales y sus necesidades. Para ello, desde el punto de vista de la investigación, a la tradicional misión de creación de conocimiento básico debe añadirse como eje fundamental la transferencia social del mismo. Así, la universidad ha de abordar como tarea prioritaria la concreción de las vías para la aplicación del conocimiento científico en la resolución de los problemas concretos que nos afectan como ciudadanía, y en particular en la transformación de nuestro tejido productivo y social, estableciendo para ello mecanismos para que la transferencia de conocimiento sea de dominio público y de pública disponibilidad, facilitando una permanente interacción entre los problemas sociales, ambientales, culturales, laborales, políticos y el trabajo de la comunidad universitaria. 
Por otro lado, la institución debe seguir progresando hacia la universalización del conocimiento, incorporando también la divulgación de los progresos científicos y tecnológicos de forma libre como parte del compromiso social de la universidad. Para ello, debe involucrarse en los debates de actualidad social, aclarando los fundamentos científicos sobre los que deben basarse cuestiones tales como la crisis económica, el cambio climático o la teoría de la evolución. La universidad es un lugar privilegiado para trabajar estos problemas sociales y elaborar una producción científica cada vez mayor sobre temáticas transversales tales como el decrecimiento, la igualdad de género o la cultura de la paz.

Como garantía del cumplimiento de tales objetivos, es necesario establecer los mecanismos para que en la universidad haya una autoevaluación y coevaluación permanente, no planteada como rendición de cuentas en el sentido de "auditoría contable" para establecer ordenaciones y rankings, sino como revisión y mejora de sus actuaciones, tanto institucional como individual, y exigencia de un adecuado aprovechamiento de los recursos públicos puestos a su disposición. Una evaluación que debe incluir indicadores del impacto social del trabajo en la universidad y de la transferencia de conocimiento que tenga utilidad social para la comunidad.

Igualmente, la actividad científica y tecnológica, desarrollada en el ámbito de la Educación Superior, debe ser elemento de cohesión social y territorial, base de un sistema científico-técnico que sustente un modelo económico y social ecológicamente sostenible y decreciente en el saqueo de recursos naturales y del ecosistema, así como la orientación de los recursos de I+D a aplicaciones civiles, descartando el modelo de desarrollo vía "innovación militar". Lo cual supone la eliminación de los gastos de construcción de armas y la aprobación de una Ley de Objeción Científica que legalice la creciente oposición de los científicos españoles a trabajar en programas de Defensa.

Por el contrario, se tendría que promocionar la investigación asociada a modelos de economía y ecología sostenible, protección del medioambiente, energías renovables y limpias y salud laboral. Así como la puesta en marcha de líneas de ayudas financieras para el desarrollo de proyectos de investigación que respondan a las demandas de las organizaciones sociales: sindicatos, asociaciones de vecinos, consumidores, ONGs, organizaciones ecologistas, pacifistas, etc. Recuperando y reforzando la investigación en temas socioeconómicos, con especial énfasis en los objetivos dirigidos al estudio de los fenómenos de marginación y exclusión social, emigración, consecuencias sociales del paro y la precariedad laboral. Evaluación del impacto social de las políticas de reducción del gasto público y de desregulación de las condiciones de trabajo. Evaluación del impacto de las tecnologías generadas sobre el empleo, la calidad de vida y el medio ambiente.

Así como salvaguardar la investigación como servicio público, rechazando la apropiación privada de recursos públicos. Es decir, que los resultados de la investigación científica y tecnológica subvencionada con fondos públicos deben ser de libre utilización y difusión. Desarrollando tecnologías genéricas y respetuosas con el medio ambiente, que permitan mejorar la calidad de vida en los entornos urbanos y rurales.

Un modelo de I+D que aumente sustancialmente los recursos de forma efectiva y garantizada (se cifra en un mínimo del $2 \%$ del PIB) y que vaya acompañado de un Plan de reducción de la precariedad del empleo en la investigación (casi la mitad de sus efectivos con contratos temporales) y de un sistema de acreditación del profesorado universitario que supongan una evaluación más justa y que responda a un modelo de ciencia y docencia para el bien común siguiendo las propuestas de ciencia abierta u Open Science, de la Declaración de San Francisco sobre Evaluación de la Investigación (DORA) o la Declaración de Berlín.

En definitiva, lo que queremos es una Universidad y una Investigación orientada hacia intereses sociales generales: la preservación del planeta y sus recursos naturales, la paz, la igualdad de género, la economía para el bien común y la justicia social. La consecución de este modelo de universidad e investigación para el bien común requiere la recomposición del movimiento universitario, en pos de un modelo social de universidad pública, que ponga el conocimiento al servicio y al alcance de toda la sociedad frente a los objetivos clasistas, privatizadores y mercantilistas de la ofensiva neoliberal, exigiendo una universidad pública de todos y todas y para todos y todas.

\section{Bibliografía}

Amigot, P. \& Martinez, L. (2015). Procesos de subjetivación en el contexto neoliberal. El caso de la evaluación del profesorado y la investigación universitaria. Revista de la Asociación de Sociología de la Educación, 8 (2), 138-155.

Anderson, M. (2001). The complex relations between the academy and the university: Views from the literatura. The Review of the Higher Education, 72, 226-246.

Barnett, R. (2001). Los límites de la competencia. El conocimiento, la educación superior y la sociedad. Barcelona: Gedisa.

Barrón, I. (2018). Primer embargo judicial por no devolver un préstamo universitario del ICO. El País. [Recuperado el 15/05/2018 de https://goo.gl/Z9ArG5]

Barsky, O. (2014). La evaluación de la calidad académica en debate: los rankings internacionales de las universidades y el rol de las revistas científicas. Buenos Aires, Argentina: Universidad Abierta Interarmericana-Teseo.

Bok, D. (2003). Universities in the Marketplace: The Commercialization of Higher Education. Princeton, N.J.: Princeton University Pres.

Buranyi, S. (2017). ¿Son los vertiginosos beneficios de la industria editorial malos para la ciencia? Sin Permiso. [Recuperado el 22/08/2017 de https://goo.gl/zusCrg]

Calella, G. (2013). La fábrica de precarios. En Fernández González, Joseba et alt. (2013). De la nueva miseria, la universidad en la crisis y la nueva rebelión estudiantil (103-122). Madrid: Akal Pensamiento crítico.

Corral, M. (2016). España, el país europeo que más ha recortado en I+D durante la crisis. El Mundo, 10 de febrero de 2016. [Recuperado el 9/08/2017 de https://goo.gl/sPrxcs]

Delapierre, F. (2015). La bomba de la deuda estudiantil. Barcelona: Icaria.

Ellacuría, I. (1999). Escritos universitarios. San Salvador: UCA Editores.

Fernández, J. \& Valdés, V. (2015). Deuda, precariedad y flexibilidad: la nueva condición estudiantil en España. En F. Delapierre. La bomba de la deuda estudiantil (67-100). Barcelona: Icaria.

Fernández De Rota, A. (2009). Universidad, neoliberalismo y capitalismo creativo. Nómadas. Revista Crítica de Ciencias Sociales y Jurídicas, 21(1), 1-15.

Fernández González, J.; Urbán Crespo, M. \& Sevilla Alonso, C. (Coords.). (2013). De la nueva miseria. La Universidad en crisis y la nueva rebelión estudiantil. Madrid: Akal.

Flores, J.e. \& Villaseñor, J.I. (2015). Perspectivas actuales sobre los rankings mundiales de universidades. Revista de Educación Superior, 44 (175), 44-67. 
Foucault, M. (1975). Vigilar y Castigar. México: Ediciones Siglo XXI.

Foucault, M. (2004). Naissance de la biopolitique. Cours au Collège de France (1978-1979). Paris: Seuil/Gallimard.

Garzón, A. (2015). Prólogo. En F. Delapierre. La bomba de la deuda estudiantil (5-10). Barcelona: Icaria.

Gould, E. (2003). The University in a Corporate Culture. New Haven, Conn.: Yale University Pres.

Harvey, D. (2014). Guía de El Capital de Marx. Libro primero. Madrid: Akal.

Hirtt, N. (2003). Los nuevos amos de la escuela. El negocio de la enseñanza. Madrid: Minor.

Johnson, B.; Kabanagh, P. \& Mattson, K. (Eds.). (2003). Stal This University: The Rise of the Corporate University and the Academic Labour Movement. Nueva York: Routledge.

Jorge, A. \& De Frutos, R. (2016-2017). La evaluación de la investigación universitaria en España. Chasqui. Revista Latinoamericana de Comunicación, 133, 159-172.

Laval, C. (2004). La escuela no es una empresa. El ataque neoliberal a la enseñanza pública. Barcelona: Paidós.

Manzano, V. (2011). La Universidad Comprometida. Barcelona: Hipatia.

Martínez García, J.S. (2017a). El esfuerzo y el talento como ideología. Eldiario.es [Recuperado el 06/12/2017 de https://goo.gl/ z8yyQh]

Massé Narváez, C.E. (2014). Hacia una identidad crítica del universitario con miras a confrontar al estado neoliberal. Revista de antropología experimental, 14, 419-429.

Mediavilla, D. (2017). El viejo truco del Gobierno para ocultar sus recortes en ciencia. El País, 5 de abril de 2017. [Recuperado el 9/08/2017 de https://goo.gl/iJvgK]

Mirowski, Ph. (2011). Science-Mart. Privatizing American Science. Cambridge, MA: Harvard University Press.

Molero, J. \& De No, J. (2015). Informe COSCE: Análisis de los recursos destinados a I+D+i (Política de Gasto 46) contenidos en los Presupuestos Generales del Estado Aprobados para el año 2015. [Recuperado el 9/08/2017 de https://goo.gl/wGvW7M]

Monbiot, G. (2017). ¿Cómo nos metimos en este desastre? Madrid: Sexto Piso.

Moya, J.; Pérez-Espona, S.; Hortal, J.; Valladares, F.; Rodriguez-Gironés, M.a.; Santamaría, L. \& Montserrat, M. (2017). El flujo de conocimiento de lo público a lo privado: ¿No hay canon? El Diario.es [Recuperado el 2/10/2017 de https:/goo. $\mathrm{gl} / \mathrm{j} 5 \mathrm{rVze}]$

Munárriz, A. (2017). Dos multinacionales controlan el negocio de los artículos científicos en España. Infolibre. [Recuperado el 9/08/2017 de https://goo.gl/S5F53m]
Nichols, S. N. \& Berliner, D. C. (2007). Collateral Damage: The effects of high-stakes testing on America's schools. Cambridge, MA: Harvard Education Press.

PIneda, P. \& Celis, J. (2017). ¿Hacia la universidad corporativa? Reformas basadas en el mercado e isomorfismo institucional en Colombia. Archivos Analíticos de Politicas Educativas=Education Policy Analysis Archives, 25(1), 1-19.

Ramió, C. (2014). Manual para los atribulados profesores universitarios. Madrid: Catarata.

Readings, W. (1996). The University in Ruins. Cambridge, Mas.: Harvard University Pres.

Rhoades, G. \& Slaughter, S. (2010). Capitalismo académico en la nueva economía. Retos y decisiones. Pasajes: Revista de pensamiento contemporáneo, 33(14), 43-59.

Rodríguez Victoriano, J.M. (2017). La praxis de la excelencia universitaria entre la paranoia de sus promotores y la culpa de sus víctimas: hacia la recuperación del deseo docente y la universidad pública. Teknokultura. 14 (1), 85-103.

Rushkoff, D. (2000). Coerción. Por qué hacemos caso a lo que nos dicen. Barcelona: Círculo de Lectores.

Saltelli, A. (2017). "La ciencia hoy es una mercancía". CTXT E Observatorio Social de la Caixa. [Recuperado el 6/08/2017 de https://goo.gl/F1mqp3]

Sampedro, J. (2017). Logro científico. El País. [Recuperado el 21/12/2017 de https://goo.gl/cEZ9Lf]

Sevilla, C. (2010). La fábrica del conocimiento. La Universidad-empresa en la producción flexible. Madrid: El Viejo Topo.

Slaughter, S. \& Leslie, L.L. (1997). Academic Capitalism: Politics, Policies and the Entrepreneurial University. Baltimore and London: The John Hopkins University Press.

Slaughter, S. \& Rhoades G. (2004). Academic Capitalism in The New Economy. Baltimore: The John Hopkins University Press.

Thrift, N. (2017). Elite universities strive for inclusivity - but only up to a point. Times Higher Education. [Recuperado el 13/11/2017 de https://goo.gl/6XascD]

Vila, E. (2012). Ciudadanía, equidad e innovación: reflexiones sobre la política de responsabilidad social de las universidades. Innovación Educativa, 12 (59), 61-85.

Villareal, A. \& Escudero, J. (2018). Todos contra Elsevier, el gigante editorial científico que cobra a España 25 'kilos' al año. El Confidencial. [Recuperado de https://goo.gl/iBkU8K el 15 de febrero de 2018] 
